\title{
Digital Camouflage Images Using Two-scale Decomposition
}

\author{
Hui Du ${ }^{1,2}$, Xiaogang $\operatorname{Jin}^{1}$, Xiaoyang $\mathrm{Mao}^{3}$ \\ ${ }^{1}$ State Key Lab of CAD\&CG, Zhejiang University, China \\ ${ }^{2}$ Zhejiang University of Media and Communications, China \\ ${ }^{3}$ University of Yamanashi, Japan
}

\begin{abstract}
We present an alternative approach to create digital camouflage images which follows human's perception intuition and complies with the physical creation procedure of artists. Our method is based on a two-scale decomposition scheme of the input images. We modify the large-scale layer of the background image by considering structural importance based on energy optimization and the detail layer by controlling its spatial variation. A gradient correction is presented to prevent halo artifacts. Users can control the difficulty level of perceiving the camouflage effect through a few parameters. Our camouflage images are natural and have less long coherent edges in the hidden region. Experimental results show that our algorithm yields visually pleasing camouflage images.
\end{abstract}

Categories and Subject Descriptors (according to ACM CCS): I.4.9 [Image Processing and Computer Vision]: Applications-

\section{Introduction}

Optical illusions (also called visual illusions) create images perceived in a manner that differs from objective reality. As a form of cognitive illusions, camouflage images (also called hidden images) aim to demonstrate the amazing capability of human's visual system to transform visual input into interpretable shape. Through carefully placed objects, characters, coloring and shadows, foreground objects are cleverly concealed within the background scene. People usually have difficulty in recognizing objects from their backgrounds immediately when they view a camouflage image.

It is not easy to create interesting camouflage images, which are usually created by highly specialized skilled artists. Artists first sketch background and foreground objects and draw them as a coherent whole, then details are carefully added in order to achieve a balance between hiding and revealing the object [TZHM11]. However, Such a manual creation procedure is very time-consuming and is out of reach for most casual users. To address this problem, recent work $\left[\mathrm{CHM}^{*} 10\right.$, TZHM11] explicitly creates camouflage images using natural photos and produces visually pleasing results. However, when the luminance contrast of the foreground object is fairly low, these approaches cannot guarantee the consistency of luminance between the foreground and background. As a result, obvious strong long edges will emerge and they give important visual clues to the concealed objects. The approach of [TZHM11] may lead to blurred details in the results, because both the large scale structural information and the texture detail are blended in the same way. Moreover, the structural importance of the object is not taken into account [TZHM11].

As shown in Figure 1, many hidden objects have coherent textures and consistent colors with surroundings while clearly representing the structural feature of the object shape. For most objects, edges in images contain important structural information about objects and the contours representing the shape of the object are strong edges. Based on the observation, our approach utilizes edges as the structural unit in the camouflage image. The importance of edges is considered using the Gaussian scale space analysis to ensure the structures of the objects are preserved through camouflage manipulations. Perception research [BM03, EM98] shows that long coherent-edges, even when faint, are perceptually salient to the human visual system (HVS). Motivated by the law of closure of Gestalt psychology's theory, which states that humans perceive objects as a whole even when they are not complete, we take long and important edges of the object into account, and divide these edges into shorter edges and remove some of them. The viewer can still recognize the hidden object based on the remained shorter edges.

In this paper, we propose an alternative method to create camouflage images by taking the above observations into ac- 


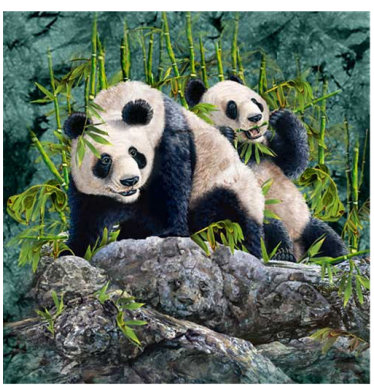

(a)

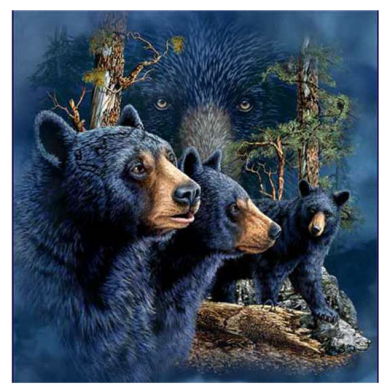

(b)
Figure 1: Camouflage images by Steven Michael Gardner. (a) 9 pandas; (b) 11 bears.

count. Given a background image and a foreground object to be hidden, the camouflage image generating process is defined as an image blending problem, which is substantially an optimization problem. The hidden object should contain coherent textures and consistent colors with surroundings while preserving the structure of the foreground object in the blending region. Inspired by the physical creation procedure of artists, we employ a two-scale computation mechanism. Such a scheme follows the intuition that the overall shape of an object is defined by its large features, and the details are defined by its small features [LMJH*11]. Our approach starts by decomposing the foreground object and the background image into the spatial structure layer and the detail layer respectively, which are modified in different ways. The process of modifying the spatial structure layers is defined as an optimization problem. Detail layers are weight-added in a way preserving the spatial location of edges in the structure layers.

A new alternative framework of digital camouflage images is proposed. Specifically, our paper makes the following contributions: (1) A novel technique for large-scale image blending: we control the large-scale spatial intensity over the result using a novel edge-aware energy minimization. (2) A fashion for managing spatial detail variation: we manipulate the intensity of the high-frequency detail and its spatial variation while respecting the intensity of large-scale layers. (3) A constraint for avoiding halo artifacts: we employ a gradient correction constraint to prevent halo artifacts.

\section{Related Work}

Many previous work has addressed how to automatically design and create optical illusion images using computational tools in computer graphics community. Oliva et al. [OTS06] develop a technique to produce static hybrid images by blending images of different frequencies. Then different images can be perceived as the viewing distance changes. Kim et al. [KP02] propose Jigsaw image mosaic that image tiles of arbitrary shape are used to compose the final picture. Orchard et al. [OK08] propose an approach to obtain higher quality mosaics by incorporating a number of improved algorithms. Genuine image mosaics are created using the method proposed by Pavić et al. [PCK09]. Their method splits an input image into a set of tiles and each tile is replaced by another image from a large database. More details about digital mosaics are provided by Battiato et al. [BBFG07].

Camouflage images are considered as a form of optical illusion art. Yoon et al. [YLK08] present a hidden picture puzzle generator which hides transformed versions of objects using a rotation-invariant shape context matching. Mitra et al. [MCL $\left.{ }^{*} 09\right]$ present a synthesis technique to generate emerging images from a given scene. Wu et al. [WFY*10] use an optimization approach for modeling and rendering impossible figures. Given a set of 3D locally possible parts of a figure, their approach optimizes a view-dependent 3D model and renders new views of impossible figures at the desired novel viewpoint. Chu et al. [CHM*10] use a texture synthesis technique to create camouflage images that have natural appearance. Their approach leaves an appropriate degree of clues for human to detect in the final result by considering object segments and their topological relations. Tong et al. [TZHM11] propose a system for creating hidden images. Their approach first finds the place where an object will be embedded within the scene using shape matching by considering object contour and edge information, then uses a modified Poisson blending approach to generate the resulting hidden image. Our approach also uses an image blending approach to create camouflage results. However, we introduce a data term for measuring the background information to the original Poisson blending and formulate the problem as a new optimization equation. Moreover, by considering the structural importance of the object, we use fewer noncoherent edges and less silhouette information to provide an appropriate degree of cues for recognizing the object while producing similar effect of subject contours.

Our work also relates to image blending which embeds an object from a source image into a target image. Alpha blending is a classical method of image blending, which usually need an accurate alpha matte of the object in order to obtain a high quality blending result. However, artifacts will arise when different illumination conditions exist between the source and target images. Gradient-based image blending methods, which are originally introduced by Pérez et al. [PGB03], usually produce seamless cloning results by solving a Poisson equation. Jia et al. [JSTS06] improve the gradient domain method by optimizing the region boundary. Chen et al. [CCT*09] introduce a hybrid image compositing method which combines Poisson blending with alpha blending. A similar result to the original Poisson blending is obtained by using mean value cloning proposed by Farbman et al. [FHL*09]. Their method uses mean value coordinates instead of solving Poisson equations for image cloning. Tao et al. [TJP10] propose an error-tolerant cloning approach 


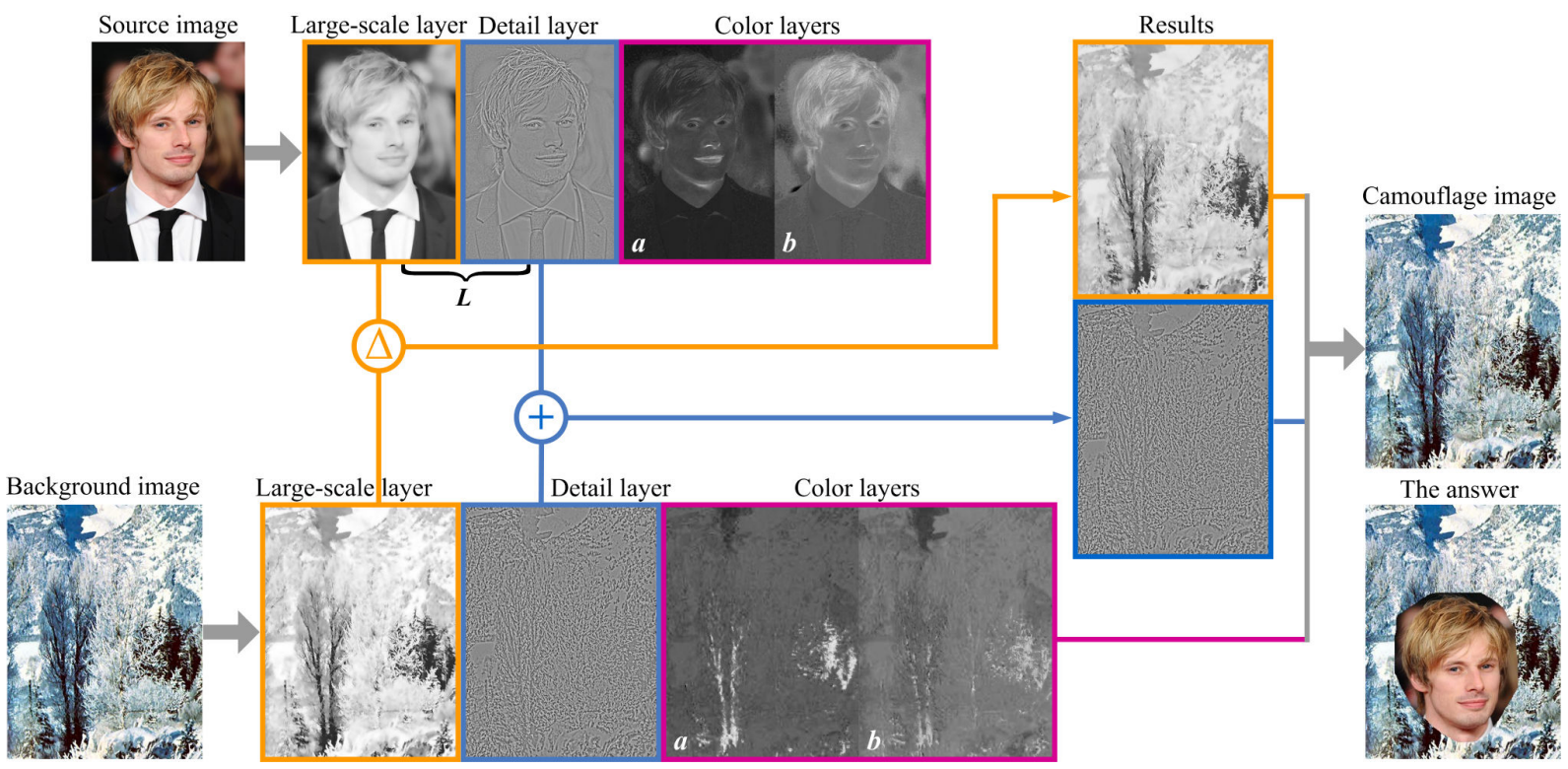

Figure 2: The flowchart of our approach. $\Delta$ denotes our large-scale nonlinear blending. + denotes weighted addition. Our approach consists of four main steps: layer decomposition, nonlinear blending for the large-scale layer, detail management and layer composition. The answer is shown on the bottom right.

which can prevent color bleeding artifacts without changing the boundary location. Sunkavalli et al. [SJMP10] reconstruct blending images from a large set of filter outputs and integrate harmonization into the composite result. Ding et al. [DT10] present a content-aware image blending method by combining combining alpha matting with Poisson methods. Xie et al. [XSMC10] propose an optimized mean value cloning method to eliminate discoloration artifacts. Zhang et al. [ZT11] improve the gradient-based method by taking the global feature of the target scene into account.

\section{Synthesis of Camouflage Images}

Vision science research shows that the luminance of an image provides sufficient visual clues for identifying the objects of a scene [Pal99]. Therefore, we focus on the process of the luminance channel of the image.

Now we explain our camouflage image generating algorithm in detail. Camouflage effect has two goals. (1). Retaining the elements of the original scene of the background image. (2). Inserting the feature of the foreground object into the final result. To reach the goal, our approach makes a compromise between retaining texture information from the background image and applying the texture from the source image consisting of the foreground object. To achieve a perceptually plausible result, we consider the preservation of the foreground object's structure and the background image's texture. This can be formulated as an optimization problem by minimizing an energy function in terms of least- squared error. The workflow of our approach is illustrated in Figure 2. A user first chooses a region by painting strokes or using Lazy Snapping method [LSTS04] to obtain the object in the source image. The chosen region or the segmented object is then automatically embedded into the background image using the algorithm.

\subsection{Layer Decomposition}

CIELAB color space is approximately perceptually uniform and better to separate lightness from color than other color spaces. Therefore, our algorithm first decomposes the foreground object and the background image into lightness and chroma channels by converting them into CIELAB color space. Then, the lightness channels of the object and the background image are decomposed to the large-scale and detail layers by applying an edge-preserving filter operator [GO11]. The large-scale layer (structure layer) denoting large features carries low frequency information of the image. The detail layer denoting small features carries high frequency information of the image. The detail layer is generated by subtracting the large-scale layer from the lightness channel.

\subsection{Large-Scale Layer Blending}

Camouflage images are created by blending foreground objects with surrounding. That is, a camouflage result should be very similar to the background image on texture and color 


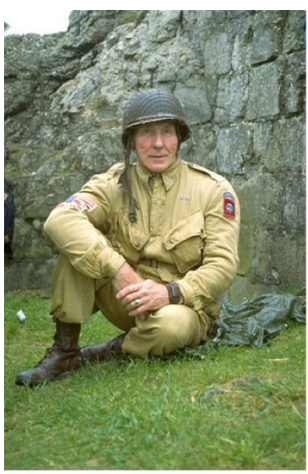

(a)

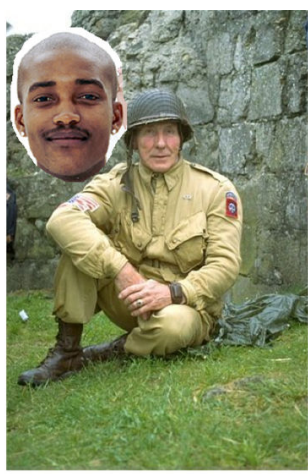

(b)

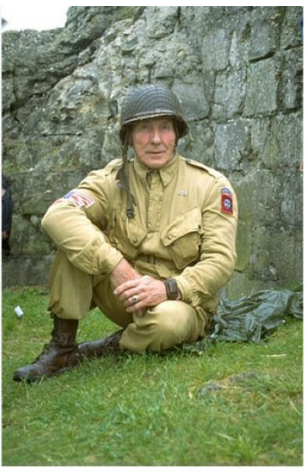

(c)

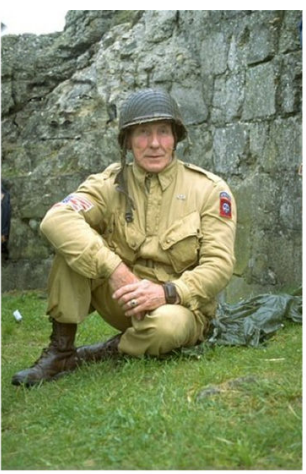

(d)

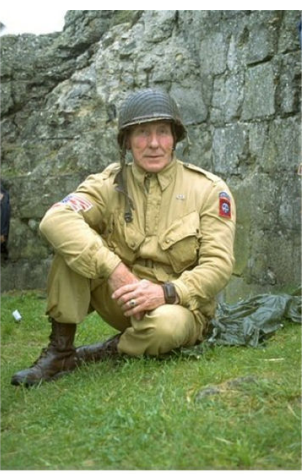

(e)

Figure 3: Comparisons using different parameter constraints. (a) The background image; (b) the foreground object; (c) the result without edge and gradient constraints (the same $\omega=0.05$ for all the pixels in the object region), which leads to obvious structural clues for recognizing; $(d)$ the result of only using edge constraints $(\kappa=0.07$, one fourth shorter edges are removed), which produces halo artifacts near strong edges; (e) the result of using both edge and gradient constraints $(\beta=0.1)$. In this example, the detail parameter $\alpha$ is set to 0 .

while preserving some structural information of the foreground object for human recognition. To preserve the important structure of the foreground object, we use a novel blending approach for the large-scale layer. Specifically, our large-scale layer blending algorithm is considered as an optimization problem by minimizing an energy function in terms of least-squared error:

$$
\begin{array}{r}
\sum_{p \in \Omega} \omega(F(p)-T(p))^{2}+(1-\omega)|\nabla F(p)-\mu \cdot g(p)|^{2} \\
\text { with }\left.F\right|_{\partial \Omega}=\left.T\right|_{\partial \Omega}
\end{array}
$$

where $F$ and $T$ represent the large-scale layer of the resulting image and the background image, respectively. $\Omega$ is the hidden region specified by the user and $\partial \Omega$ is the boundary of the region. $\nabla$ denotes a gradient operator. $\omega$ is used to control the hidden level of the foreground object.The specification of $\omega$ will be described in Section 3.3. The resulting $F$ is more similar to the background $T$ when we increase the value of $\omega$, which leads to more difficulties in recognizing the object. $\mu$ is a gradient constraint which is used to prevent halo artifacts. $g$ is a gradient field defined as:

$$
g= \begin{cases}\nabla T, & \text { if }|\nabla T|>|\nabla S| \\ \nabla S, & \text { otherwise }\end{cases}
$$

where $\nabla T$ and $\nabla S$ denote the gradient fields of large-scale layers of the background image and the foreground object in the hidden region respectively. Note that we solve the optimization for the large-scale layer only.

Unlike the standard mixing gradient cloning problem [PGB03], we add a data function term to the optimization functional. According to the Euler-Lagrange equation, we can rewrite equation 1 so that the minimization problem can be defined as the solution of the following linear system:

$$
\omega F-(1-\omega) \Delta F=\omega T-(1-\omega) \mu \nabla \cdot g
$$

where $\Delta$ is the Laplacian operator. Interestingly, equation 3 is a screened Poisson equation. This hints that we can use the method of Farbman et al. [FFL11] to solve the equation.

\subsection{Structural Edge Constraint}

The unified hidden level of the object is achieved by setting the same $\omega$ for all the pixels in the region. To perceive the foreground object, some structural contours of the object must be preserved in the camouflage result. Our approach identifies the relevant image structure represented by a hierarchy of edges in the Gaussian scale space, which has been developed by computer vision community to deal with structure identification in images with no priori information. We start from using a Canny edge detector to find the edges of the object. However, these edges have no concept of edge importance. We therefore compute the lifetime of each edge pixel in the Gaussian scale space [OBBT07] and use the lifetime as a measure of structural importance. The edges with larger lifetime correspond to more stable and important structures. We obtain a binary mask $M$ which indicates important structural edge locations of the object based on a certain lifetime threshold. As a result, we can manipulate edges in a structure-aware way.

Another concern is to deal with edge lengths. Perception studies [BM03, EM98] show that long coherent-edges are perceptually salient to the HVS even they are faint. Long edges may reveal the important information about the object. As shown in Figure 3(c) and Figure 3(d), many long edges in the results provide too many obvious visual clues. In order to better hide the foreground object, the process for long edges 
must be considered. Our main idea is to divide long and important edges of the object into smaller parts and remove some of the parts. According to the law of closure of Gestalt psychology's grouping theory, a viewer will fill in the gaps between unconnected edges and analyze scenes as a whole rather than as a set of unconnected features. Specifically, the algorithm first collects the edges longer than a certain threshold according to the mask $M$. Here we use 30 pixels as the threshold. Then the algorithm dilates these collected edges using a $5 \times 5$ window, casts some sample points on the collected long edges randomly and divides dilated long edge patches into shorter edges patches $E_{i}$ in terms of the sample points. We expect that some of shorter edges will not emerge in the hidden result. The algorithm randomly hits a percentage (specified by the user, default 20\%) of shorter edges. In addition, we also assure $\omega$ changes smoothly over each dilated edge. Thus, we define $\omega$ as

$$
\omega(p)= \begin{cases}\max \left(\kappa+(1-\kappa) e^{\frac{-(p-q)^{2}}{2 \sigma^{2}}}\right), & \text { if } E_{i} \text { is hit } \\ \max \left(\kappa\left(1-e^{\frac{-(p-q)^{2}}{2 \sigma^{2}}}\right)\right), & \text { otherwise }\end{cases}
$$

where $p$ indexes the pixel over each dilated edge patch, $q$ indexes the pixel at the corresponding chosen shorter edge in the dilated edge patch; $\kappa$ denotes a value specified by the user. The value of $\sigma^{2}$ is set to $\min ($ width, height $) / 25$ where width and height denote the width and the height of the source image respectively. After this step, we break long edges and give viewers less structural clues for identifying the object. However, the viewer can still identify the object using their imagination although parts of important edges and silhouettes are absent in the final result.

\subsection{Gradient Constraint}

As shown in Figure 3(d), applying the mixing gradient of the object and background image naively on the optimization leads to halo artifacts near strong edges. To avoid the artifact, we use a gradient constraint to reduce the coefficient $\mu$ according to the magnitudes of the gradients to avoid halo artifacts.

Removing the halo artifacts can be modeled as a Gaussian distribution formula:

$$
\begin{gathered}
\mu(p)=\exp \left(\frac{-\beta(1-\omega(p))\|\nabla S\|^{2}}{2 \sigma_{2}^{2}}\right), \\
\sigma_{2}^{2}=\operatorname{mean}\left(\|\nabla S\|^{2}\right)_{\Omega},
\end{gathered}
$$

where mean $(\cdot)$ denotes the mean value over the hidden domain $\Omega$. The default value for $\beta$ is 0.3 . The weighting $\mu$ is low when the gradient $\| \nabla S||$ is very high, which reduces halo artifacts.

\subsection{Detail Management}

By now, we have obtained the large-scale layer $F$ of the result by solving the linear system (3). The detail information of the foreground object and the background image should also be integrated into the final result. Otherwise, the result will not be realistic due to the lack of the detail. We define the resulting detail layer $D_{R}$ as a weighted sum of the object detail $D_{S}$ and the background detail $D_{T}$, i.e.

$$
D_{R}(p)=D_{T}(p)+t(p) D_{S}(p),
$$

where $0 \leq t(p) \leq \alpha . \alpha \in[0,1]$ is a user given constant for controlling the contribution of the foreground object detail. We expect that the value of $t$ varies depending on the value of $\omega$. That is, the detail of the object should not appear in the result where the parts of the foreground object are hidden completely. Thus, we define $t$ as

$$
t(p)= \begin{cases}\alpha, & \omega(p)=\kappa \\ (1-\omega(p)) \alpha, & \text { otherwise }\end{cases}
$$

For different pixels, different $t$ values are used. Pixels with large $\omega$ values imply small $t$ values, and the resulting pixels are much closer to the background pixels.

Once the large-scale and detail layers are obtained, we sum them to obtain the resulting lightness channel. By integrating the chroma channels of the background image, we can convert them back to the RGB color space to obtain the final synthesized image to faithfully preserve the original background color.

\section{Experiments}

\subsection{Results and Discussion}

In this section, we show the results generated by our algorithm and compare our approach to previous algorithms. Our experimental environment involves a computer with a 2.67GHz CPU of Intel Core 2 Duo and 2GB memory, 32bit Windows operating system, and Matlab version 7.7. The experiments show that the camouflage results produced by our algorithm are visually pleasing. The computation time of our implementation and parameter values are shown in Table 1 . The computation of the edge importance and the solving of the linear system consumes most of the CPU time. We believe that the performance of our method can be further improved using GPU acceleration.

As stated above, the object becomes more difficult to recognize when $\kappa$ increases and the result involves more details of the foreground object when $\alpha$ increases. Experiment results show that the foreground object is too obvious for $\kappa<0.02$ and is almost completely hidden for $\kappa>0.5$. Figure 4 and Figure 6 show some results. We use different $\kappa$ values (see Table 1) to generate different difficulty levels for the objects. Note that the exact recognition of the hidden object by viewers is a subjective issue. Different viewers may have different answers for the same camouflage image if they have no prior knowledge of the objects. 
Hui Du \& Xiaogang Jin \& Xiaoyang Mao / Digital Camouflage Images Using Two-scale Decomposition
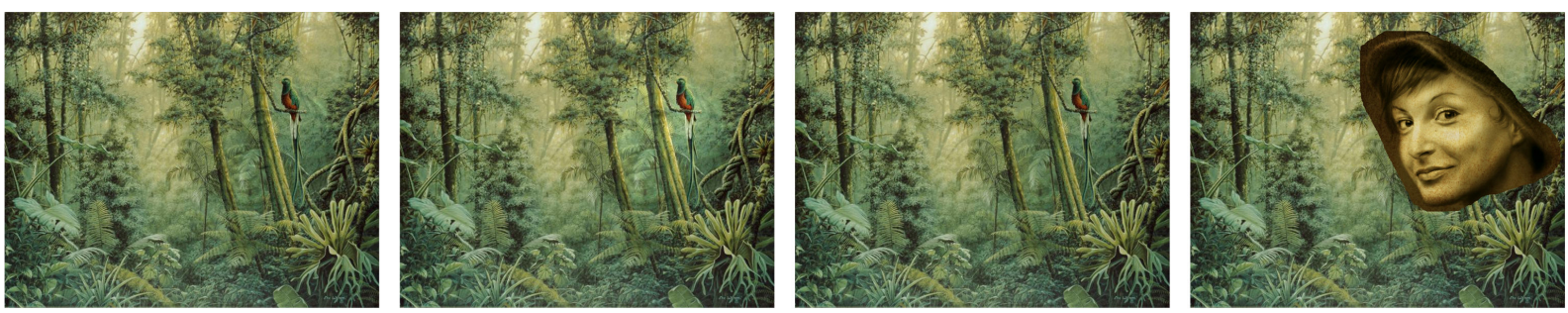

(a)
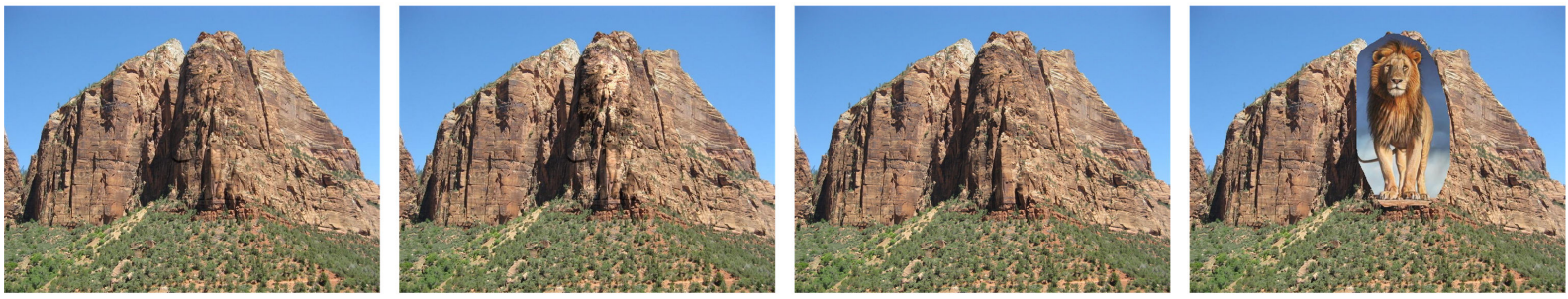

(b)
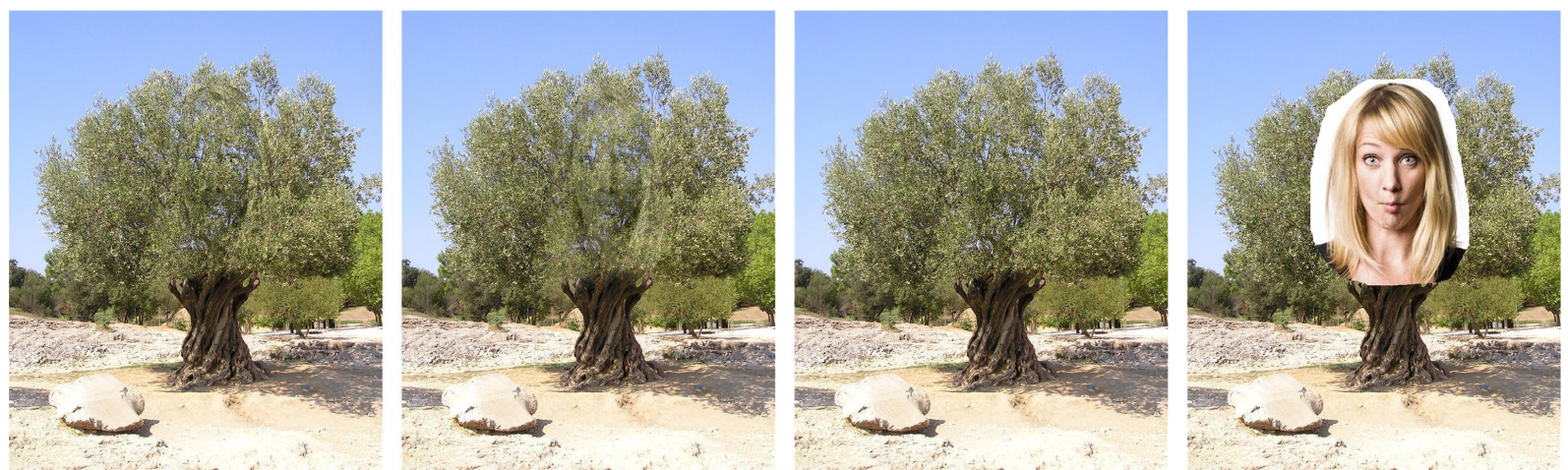

(c)
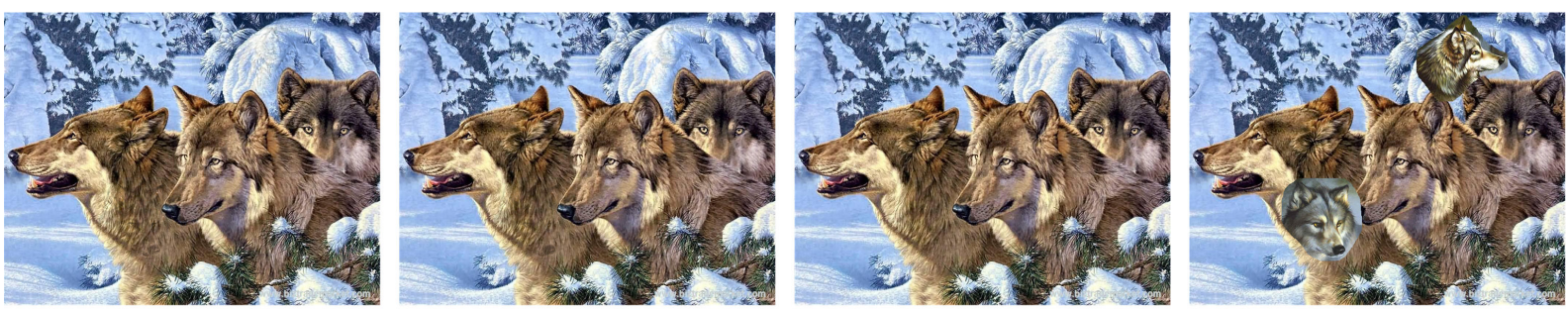

(d)
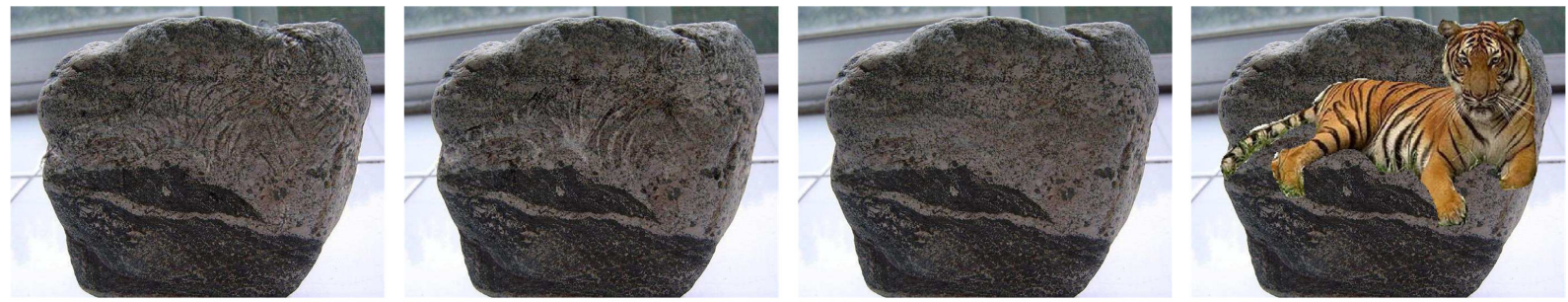

(e)

Figure 4: Comparisons with Tong et al.'s method. From left to right: our results, the results of Tong et al.'s approach, background images, the foreground objects. Please zoom in the camouflage images to better recognize the hidden objects. 


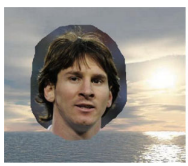

(a)

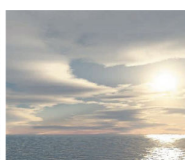

(b)

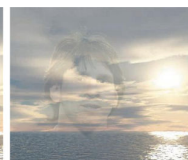

(c)

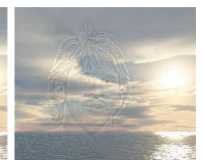

(d)
Figure 5: Seamless transparency composition. (a) The direct pasting result; $(b)$ the background image; $(c)$ the result of seamless cloning followed by alpha blending $(\alpha=0.16) ;(d)$ our result. Our result includes more detail information of the foreground region while guaranteing the transparent effect.

In Figure 4, we compare our results to those of Tong et al.'s approach [TZHM11]. Important structural features of the object are preserved since we integrate structural importance of the object into our algorithm. Therefore, we may place the foreground object on any location in the background image according to user's desire. In addition, the shape matching step becomes unnecessary in our algorithm while it is a very important step in Tong et al.'s approach [TZHM11]. In their approach, the hidden results may include obvious artifacts when matching edges are not found in the background. For instance, the luminance in the synthesized region is altered after applying their approach, which leads to luminance difference (Figure 4(a) and 4(b)). The luminance difference between the synthesized region and the background leads to unnatural hidden results and provides significant visual clues for recognizing the hidden object. As shown in Figure 4(c), the texture of the background in the region becomes fuzzy in their approach. On the contrary, our approach always guarantees that the resulting texture is realistic and coherent because of the employment of a twoscale computation scheme. From the comparisons, we can find that the advantages of our algorithm are twofold. Firstly, our approach produces natural results with coherent texture, consistent color and luminance with surroundings. Secondly, our approach is faster since the shape matching step is not used.

We also compare our results to those in Chu et al.'s method $\left[\mathrm{CHM}^{*} 10\right]$. Figure 6 shows the comparison with their approach. Our approach is quite different from their approach. Chu et al.'s method $\left[\mathrm{CHM}^{*} 10\right]$ generates new texture of the object according to the texture of the background image and the structure of the foreground object. Instead of emphasizing on the relative luminance of the regions of the hidden object, our method emphasizes on the edges of the hidden object. Our method uses different ways to process the structural layer and the detail layer by using a two-scale decomposition scheme. When the luminance contrast of the foreground object is quite low, their approach may fail. On the contrary, our approach can still generate natural hidden effects. More hidden results are shown in Figure 7.

\subsection{Applications}

While our focus is on the generation of camouflage images, we can also obtain the seamless result allowing for transparency effects. To handle seamless transparency composition, we set $\omega$ in formula 1 to a unified value and set the gradient $g$ to that of the foreground region. A single alpha value leads to obvious seam using the traditional alpha blending. In contrast, seamless results are generated since we perform the process in the gradient domain. Meanwhile, our result owns the texture information of both the foreground and background images in the composition region. Figure 5 shows an example of seamless transparency composition.

\subsection{Limitations}

Some viewers may fail to find the hidden objects in some of our hidden results. We understand that recognizing the hidden object in a camouflage image is a highly subjective issue depending on the human viewer. Our approach can generate visually pleasing hidden results at different difficulty levels in most cases. However, as shown in the top row of Figure 8, our approach may produce unsatisfactory results. In this example, the method of Tong et al. [TZHM11] generates better results with harmonious edges, since their method strengthens the background image edges matched with those of the transformed object. To sum up, our approach has the following limitations. (1) Our algorithm may fail to hide objects if the edge detection fails. In this case, the method of Chu et al. [CHM $\left.{ }^{*} 10\right]$ is a good choice since they use object segmentation and texture synthesis techniques to generate hidden images. (2) When the background image is fully occupied with strong edges, the edges of the hidden object can be overwhelmed with the textures of the background image (middle row of Figure 8). In this case, the existing methods $\left[\mathrm{CHM}^{*} 10\right.$, TZHM11] produce better results. For instance, the method of Tong et al. [TZHM11] can help the viewer to recognize the object by strengthening the matched edges within the background image. (3) Texture artifacts may occur if the texture of the hidden object is highly distinctive from that of the background image, as illustrated in the bottom row of Figure 8.

The above discussion reveals that our approach is an alternative to the previous camouflage image techniques. For different images, a user may choose an appropriate technique to generate camouflage images. For example, our approach and Tong et al.'s method are better for background images with low texture variation or for foreground objects with low constrast.

\section{Conclusions and Future Work}

We have presented an alternative perception-driven approach to generate digital camouflage images. By integrating the edge-preserving decomposition, the non-linear analysis on the large-scale layer and the linear analysis on the detail 

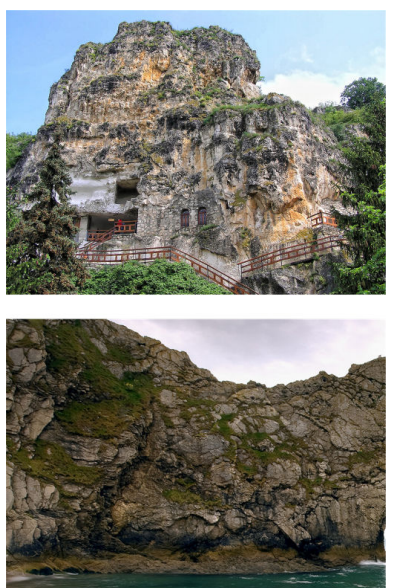

(a)
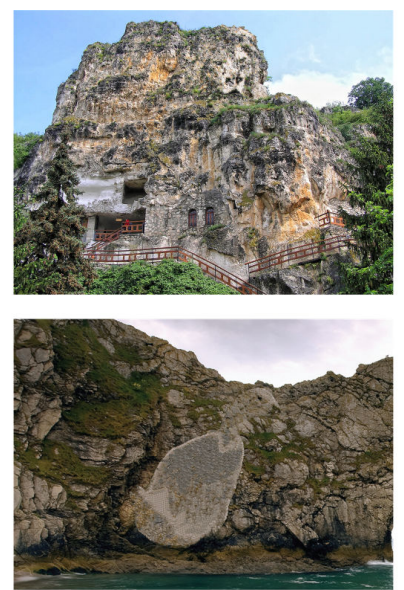

(b)
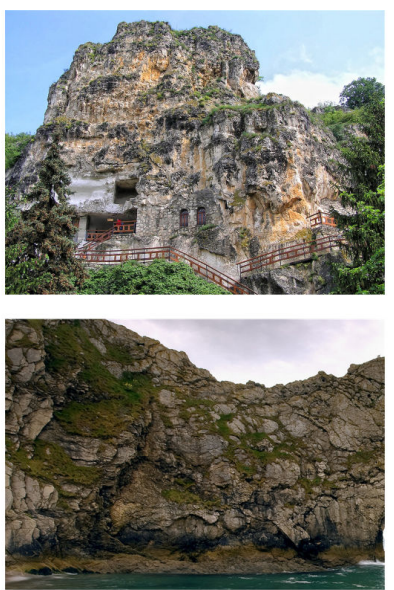

(c)
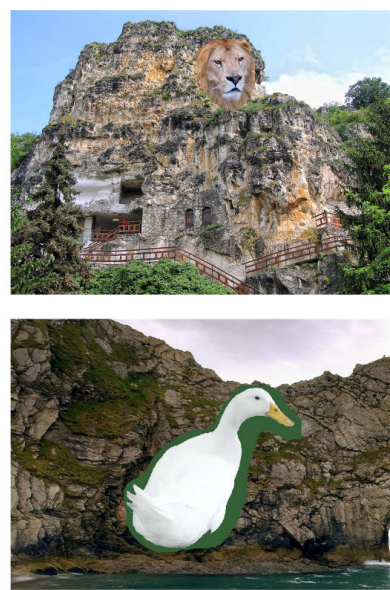

(d)

Figure 6: Comparisons with the method of Chu et al. [CHM*10]. (a) Our results; (b) the results of Chu et al.'s approach; (c)the background images; (d)the foreground objects. Please zoom in the camouflage images to better recognize the hidden objects.

Table 1: Parameter values and algorithm performance. percentage denotes the amount of shorter edges that are hit in structural edge constraints.

\begin{tabular}{|c|c|c|c|c|c|c|c|c|c|}
\hline \multirow{2}{*}{ Example } & \multirow{2}{*}{$\begin{array}{l}\text { Background } \\
\text { image }\end{array}$} & \multirow{2}{*}{$\begin{array}{l}\text { Foreground } \\
\text { object }\end{array}$} & \multicolumn{4}{|c|}{ Parameter values } & \multirow{2}{*}{$\begin{array}{l}\text { Computing } \\
\text { lifetime }(\mathrm{s})\end{array}$} & \multirow{2}{*}{$\begin{array}{l}\text { Solving } \\
\text { system (s) }\end{array}$} & \multirow{2}{*}{$\begin{array}{l}\text { Total } \\
\text { time }(\mathrm{s})\end{array}$} \\
\hline & & & $\kappa$ & $\alpha$ & $\bar{\beta}$ & percentage & & & \\
\hline Fig.3 & $358 \times 545$ & $138 \times 183$ & 0.07 & 0.0 & 0.10 & $1 / 5$ & 0.88 & 0.05 & 0.93 \\
\hline Fig.4(a) & $735 \times 559$ & $393 \times 341$ & 0.20 & 0.20 & 0.30 & $1 / 5$ & 5.02 & 0.29 & 5.31 \\
\hline Fig.4(b) & $999 \times 736$ & $226 \times 424$ & 0.10 & 0.20 & 0.10 & $1 / 5$ & 3.29 & 0.23 & 3.52 \\
\hline Fig.4(c) & $662 \times 786$ & $252 \times 353$ & 0.04 & 0.50 & 0.10 & $1 / 5$ & 2.1 & 0.25 & 2.35 \\
\hline Fig.4(d.left) & $1024 \times 768$ & $219 \times 217$ & 0.05 & 0.50 & 0.15 & $1 / 3$ & 1.76 & 0.09 & 1.85 \\
\hline Fig.4(d.right) & $1024 \times 768$ & $249 \times 226$ & 0.20 & 0.00 & 0.60 & $1 / 3$ & 2.08 & 0.11 & 2.19 \\
\hline Fig.4(e) & $680 \times 510$ & $606 \times 383$ & 0.10 & 0.00 & 0.30 & none & 8.40 & 0.53 & 8.93 \\
\hline Fig.7(a) & $1024 \times 768$ & $254 \times 278$ & 0.10 & 0.30 & 0.15 & none & 1.7 & 0.18 & 2.88 \\
\hline Fig.7(b) & $564 \times 585$ & $174 \times 231$ & 0.05 & 0.40 & 0.30 & $1 / 5$ & 1.53 & 0.10 & 1.63 \\
\hline Fig.7(c) & $996 \times 742$ & $354 \times 442$ & 0.30 & 0.0 & 0.15 & $1 / 5$ & 3.74 & 0.41 & 4.15 \\
\hline Fig.7(d.left) & $1024 \times 768$ & $264 \times 277$ & 0.10 & 0.15 & 0.30 & $1 / 3$ & 1.73 & 0.16 & 1.89 \\
\hline Fig.7(d.right) & $1024 \times 768$ & $313 \times 407$ & 0.15 & 0.30 & 0.30 & $1 / 3$ & 3.05 & 0.25 & 3.3 \\
\hline Fig.7(e.left) & $544 \times 401$ & $202 \times 205$ & 0.10 & 0.50 & 0.40 & $1 / 5$ & 0.93 & 0.10 & 1.03 \\
\hline Fig.7(e.right) & $544 \times 401$ & $179 \times 188$ & 0.20 & 0.30 & 0.50 & $1 / 5$ & 0.76 & 0.10 & 0.86 \\
\hline
\end{tabular}

layer, our approach can control both the large-scale structure and the detail over an image. Our approach effectively hides the foreground object, preventing it from standing out from its surroundings. Our algorithm can produce good results even with low contrast objects, which is problematic in $\left[\mathrm{CHM}^{*} 10\right]$.

Our approach uses inconsistency edges to reduce the visual cue for recognizing the object. An alternative idea is to model the subject contour in optical illusions and integrate this computational model into our camouflage approach. In our current implementation, $\alpha$ and $\kappa$ are two independent parameters. Since parameter $\alpha$ plays a role in detail blending, its correlation with $\kappa$ is worth evaluating to better un- derstand the difficulty setting. Given a foreground object, automatically finding the best hidden location can enhance a camouflage image and the degree of difficulty of camouflage image. Hence, another future work is to combine our approach with previous shape matching methods at cost of increasing computational time.

\section{Acknowledgements}

We would like to thank the anonymous reviewers for their constructive comments. This work was supported by NSFC-MSRA Joint Funding (Grant no. 60970159), Zhejiang Provincial Natural Science Foundation of China (Grant 
Hui Du \& Xiaogang Jin \& Xiaoyang Mao / Digital Camouflage Images Using Two-scale Decomposition
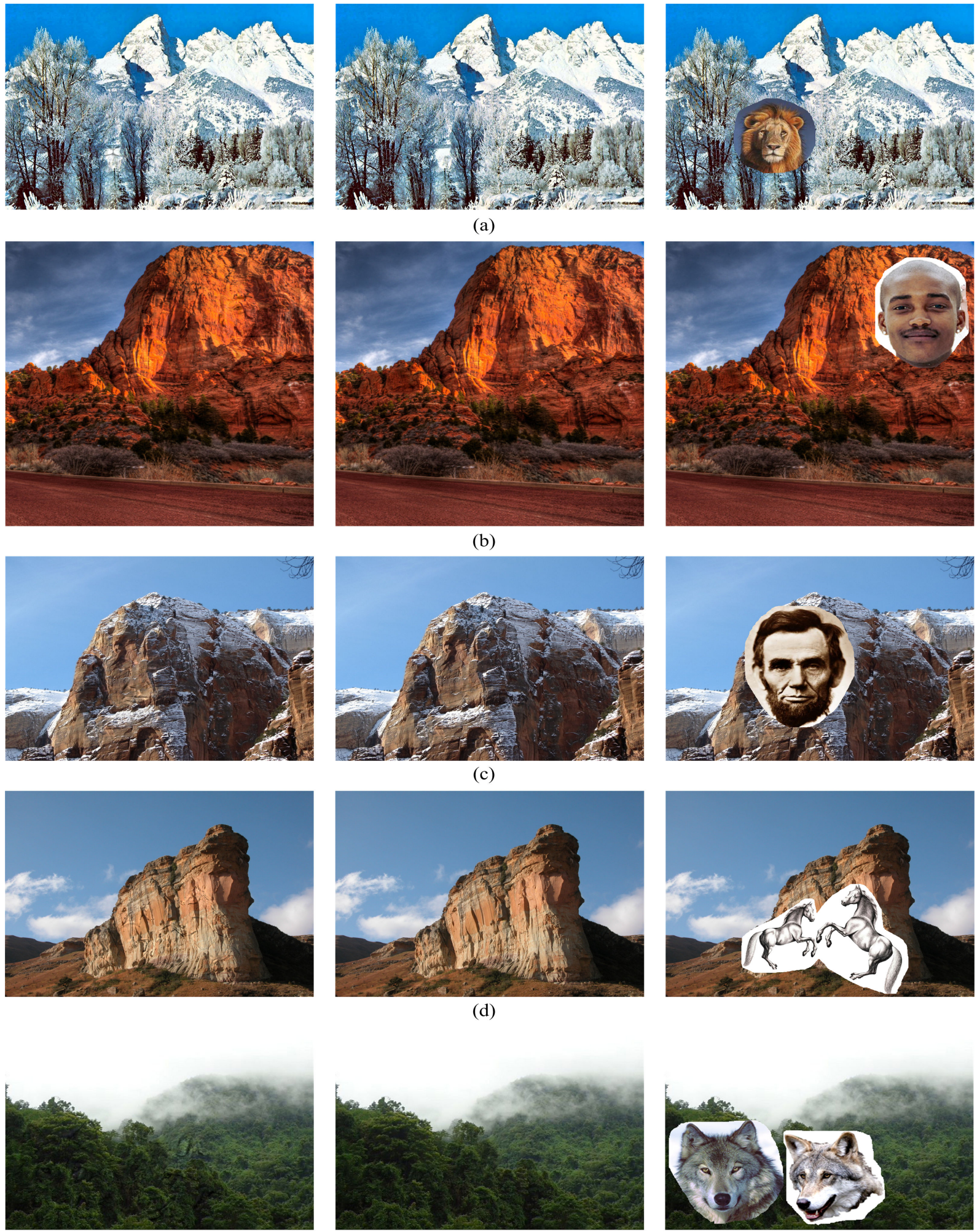

(e)

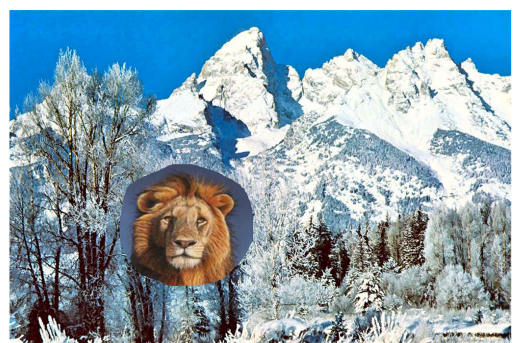

(a)
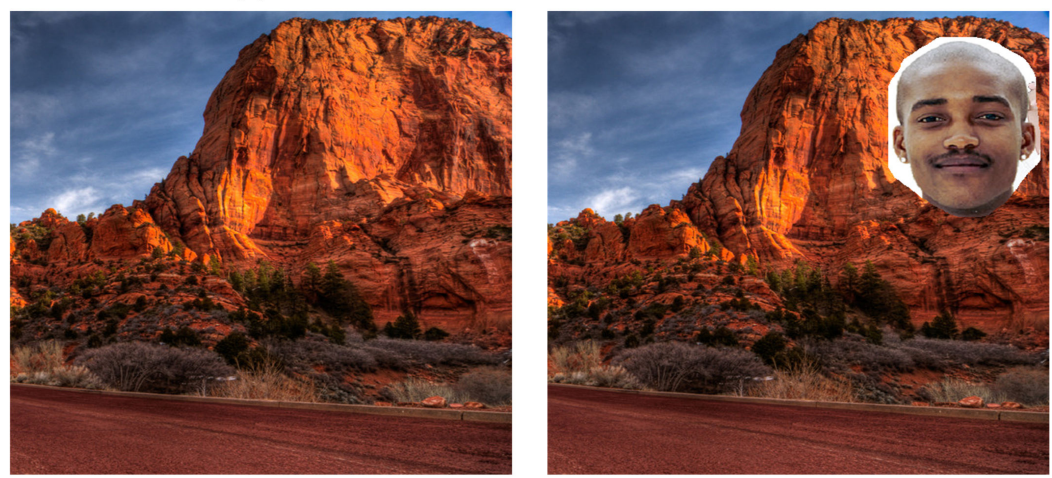

(b)

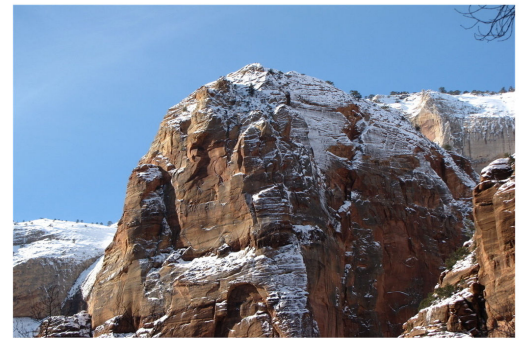

(c)

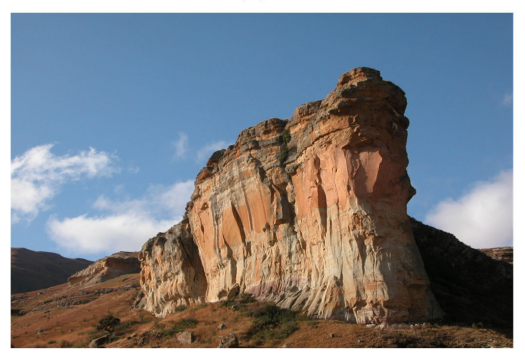

(d)
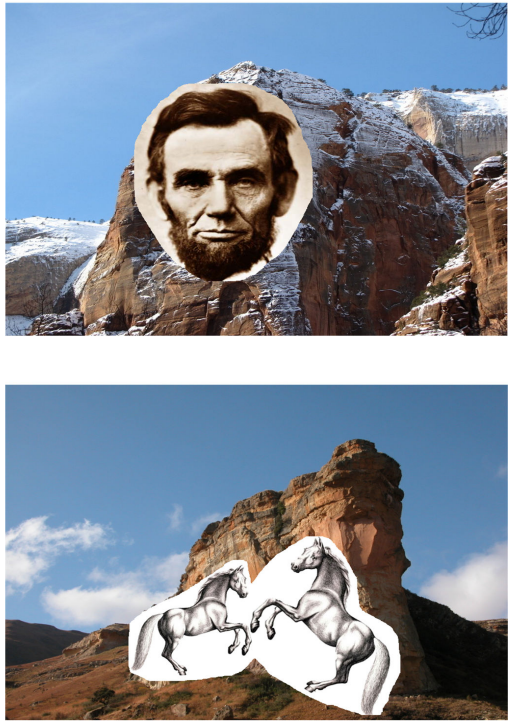

Figure 7: More results. Left: camouflage images created by our method. Middle: original background images. Right: the foreground objects. Please zoom in the camouflage images to better recognize the hidden objects.

(c) 2012 The Author(s)

(C) 2012 The Eurographics Association and Blackwell Publishing Ltd. 


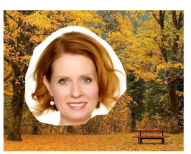

(a)

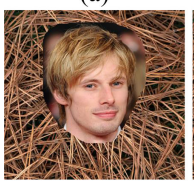

(e)

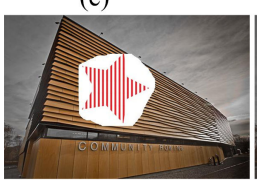

(i)

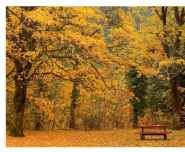

(b)

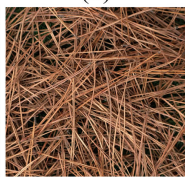

(f)

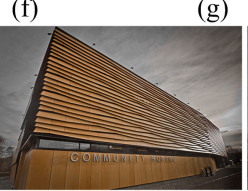

(j)

(c)

(g)
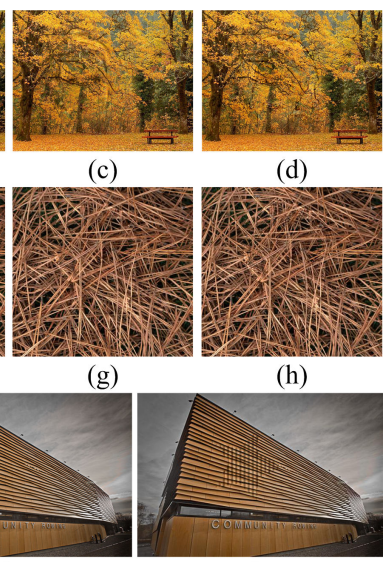

(k)
Figure 8: Limitations. Top row: (a) the foreground object; (b) the background image; (c) our method generates an unsatisfactory result; $(d)$ a better result by Tong et al.'s method. Middle row: a male head has been hidden. (e) The foreground object; $(f)$ the background image; $(g)$ the result with $\alpha=0$; $(h)$ the result with $\alpha=1$. Since the background image is fully occupied by strong edges, the structural edges of the object are completely overwhelmed with the textures of the background image. As a result, the head is almost invisible even we add the full detail of the head. Bottom row: a star with hatching patterns (i) is hidden into the background image (j) which also has hatching patterns. The texture of the star is highly distinctive from that of the background image, which leads to obvious texture artifacts with our result ( $k$ ).

no. Z1110154), and the National Natural Science Foundation of China (Grant no. 60933007).

\section{References}

[BBFG07] Battiato S., Blasi G. D., Farinella G. M., GALLO G.: Digital mosaic frameworks - an overview. Computer Graphics Forum 26, 4 (2007), 794-812. 2

[BM03] Beaudot W. H., Mullen K. T.: How long range is contour integration in human color vision? Vis Neurosci 20,1 (2003), 51-64. 1, 4

[CCT*09] Chen T., Cheng M.-M., Tan P., Shamir A., Hu S.-M.: Sketch2photo: Internet image montage. ACM Transactions on Graphics 28, 5 (2009), 124:1-124:10. 2

[CHM*10] Chu H.-K., HSU W.-H., Mitra N. J., COHEN-OR D., Wong T.-T., LEE T.-Y.: Camouflage images. ACM Transactions on Graphics 29, 4 (2010), 51:1-51:8. 1, 2, 7, 8

[DT10] Ding M., Tong R.-F.: Content-aware copying and pasting in images. The Visual Computer 26, 6-8 (2010), 721-729. 3

[EM98] ELDER J. H., M. G. R.: Image editing in the contour domain. IEEE Transactions on Pattern Analysis and Machine Intelligence 23 (1998), 291-296. 1, 4

[FFL11] Farbman Z., FATtal R., Lischinski D.: Convolution pyramids. ACM Transactions on Graphics 30 (2011), 175:1175:8. 4
[FHL*09] Farbman Z., HofFer G., LiPMAN Y., COHEN-Or D., LISCHINSKI D.: Coordinates for instant image cloning. ACM Transactions on Graphics 28, 3 (2009), 67:1-67:9. 2

[GO11] Gastal E. S. L., Oliveira M. M.: Domain transform for edge-aware image and video processing. ACM Transactions on Graphics 30, 4 (2011), 69:1-69:12. 3

[JSTS06] Jia J., Sun J., TAng C.-K., Shum H.-Y.: Drag-anddrop pasting. ACM Transactions on Graphics 25, 3 (2006), 631637. 2

[KP02] Kim J., Pellacini F.: Jigsaw image mosaics. ACM Transactions on Graphics 21, 3 (2002), 657-664. 2

[LMJH*11] LOPEZ-MORENO J., JIMENEZ J., HADAP S., REINHARD E., ANJYO K., Gutierrez D.: Non-photorealistic, depth-based image editing. Computers \& Graphics In press (2011). 2

[LSTS04] Li Y., Sun J., TANG C.-K., Shum H.-Y.: Lazy snapping. ACM Transactions on Graphics 23, 3 (2004), 303-308. 3

[MCL*09] Mitra N. J., ChU H.-K., LeE T.-Y., Wolf L., Yeshurun H., COHEN-Or D.: Emerging images. ACM Transactions on Graphics 28, 5 (2009), 163:1-163:8. 2

[OBBT07] Orzan A., Bousseau A., Barla P., Thollot J.: Structure-preserving manipulation of photographs. In International Symposium on Non-Photorealistic Animation and Rendering (NPAR) (2007). 4

[OK08] ORCHARD J., KAPLAN C. S.: Cut-out image mosaics. In NPAR '08: Proceedings of the ACM SIGGRAPH/Eurographics Symposium on Non-Photorealistic Animation and Rendering (2008), pp. 79-87. 2

[OTS06] Oliva A., Torralba A., Schyns P. G.: Hybrid images. ACM Transactions on Graphics 25, 3 (2006), 527-532. 2

[Pa199] PALMER S. E.: Vision science : photons to phenomenology. MIT Press, Cambridge, Mass., 1999. 3

[PCK09] Pavić D., Ceumern U., Kobbelt L.: Gizmos: Genuine image mosaics with adaptive tiling. Computer Graphics Forum 28, 8 (2009), 2244-2254. 2

[PGB03] PÉrez P., Gangnet M., Blake A.: Poisson image editing. ACM Transactions on Graphics 22, 3 (2003), 313-318. 2,4

[SJMP10] Sunkavalli K., Johnson M. K., Matusik W., PFISTER H.: Multi-scale image harmonization. ACM Transactions on Graphics 29, 4 (2010), 125:1-125:10. 3

[TJP10] TAO M. W., Johnson M. K., PARIS S.: Error-tolerant image compositing. In European Conference on Computer Vision (2010). 2

[TZHM11] TONG Q., ZHANG S.-H.,HU S.-M., MARTIN R. R.: Hidden images. In NPAR '11: Proceedings of the ACM SIGGRAPH/Eurographics Symposium on Non-Photorealistic Animation and Rendering (2011), pp. 27-34. 1, 2, 7

[WFY*10] WU T.-P., FU C.-W., YEUnG S.-K., JIA J., TANG C.-K.: Modeling and rendering of impossible figures. ACM Transactions on Graphics 29, 2 (2010), 13:1-13:15. 2

[XSMC10] XIE Z.-F., Shen Y., MA L.-Z., ChEn Z.-H.: Seamless video composition using optimized mean-value cloning. The Visual Computer 26, 6-8 (2010), 1123-1134. 3

[YLK08] YoON J.-C., LEE I.-K., KANG H.: A hidden-picture puzzles generator. Computer Graphics Forum 27, 7 (2008), 1869-1877. 2

[ZT11] ZhaNG Y., TONG R.: Environment-sensitive cloning in images. The Visual Computer 27, 6-8 (2011), 739-748. 3 